\title{
Resíduo de beneficiamento do granito como fertilizante alternativo na cultura do café 1
}

\author{
Granite processing waste as an alternative fertilizer for the coffee crop
}

\author{
André Guarçoni M. ${ }^{2 *}$ e César José Fanton
}

\begin{abstract}
Resumo - O resíduo de beneficiamento do granito é considerado um contaminante do ambiente. Sua utilização na agricultura como fertilizante tem sido testada e os resultados satisfatórios definidos mais por questões ideológicas do que por real eficácia. Tendo por objetivo avaliar o efeito do resíduo de beneficiamento do granito na adubação de plantas de café da espécie conilon, foi instalado um experimento em casa de vegetação, utilizando duas amostras de um mesmo solo (superficial e subsuperficial), seis doses de resíduo de beneficiamento do granito $\left(0,0 ; 1,5 ; 3,0 ; 6,0 ; 9,0\right.$ e $15,0 \mathrm{~g} \mathrm{dm}^{-3}$, equivalentes a $0 ; 3 ; 6 ; 12 ; 18$ e $\left.30 \mathrm{t} \mathrm{ha}^{-1}\right)$ e duas doses de calcário ( 0,4 e 1,2 vezes a necessidade de calagem). Após incubação das amostras, plantio, crescimento e colheita das plantas de café conilon, foram determinados teores de elementos químicos e características de planta e solo, podendo-se concluir que: a aplicação de doses crescentes do resíduo de beneficiamento do granito elevou o pH do solo e reduziu o teor de $\mathrm{Al}^{3+}$, de forma linear, mas em pequena magnitude; a utilização do resíduo de beneficiamento do granito foi mais eficiente em amostra de solo com maior capacidade tampão; para o crescimento inicial do café, o resíduo deve ser utilizado, exclusivamente, como fonte de $\mathrm{K} \mathrm{e} \mathrm{Ca}$, sendo necessário, para isso, que o $\mathrm{pH}$ do solo esteja em torno de 5,0; doses próximas a $20 \mathrm{t} \mathrm{ha}^{-1}$ de resíduo de beneficiamento do granito geraram crescimento inicial adequado para o café.
\end{abstract}

Palavras-chave - Coffea canephora. Fonte de nutrientes. Disponibilidade de nutrientes. Crescimento.

\begin{abstract}
The granite processing waste is considered an environmental contaminant. Its agricultural use has been investigated and satisfactory results have been defined basically considering ideological questions instead of its real effectiveness. Aiming to evaluate the effect of the granite processing waste on conilon coffee fertilizing, an experiment was installed under greenhouse conditions. Two samples of only one soil (superficial and subsuperficial), six granite processing waste doses $\left(0,0 ; 1,5 ; 3,0 ; 6,0 ; 9,0\right.$ and $15 \mathrm{~g} \mathrm{dm}^{-3}$, equivalent the $0 ; 3 ; 6 ; 12 ; 18$ e $\left.30 \mathrm{t} \mathrm{ha}^{-1}\right)$ and two liming levels $(0,4 \mathrm{e}$ $1,2 \mathrm{x}$ liming necessity) were investigated. After soil incubations and following the transplantation, growth and harvesting of coffee plants, have been determined contents of chemicals elements and characteristics of plant and soil. The granite processing waste application induced a linearly increase on $\mathrm{pH}$ and reduces the $\mathrm{Al}^{3+}$ contents, but in small magnitude. Granite processing waste was more efficiently when used in grater buffer capacity soil sample; for de initial coffee plants growth, it must be used, with priority, as a source of $\mathrm{K}$ and $\mathrm{Ca}$, in this case, a soil $\mathrm{pH}$ around 5.0 is recommended; doses of granite processing waste next to a 20.0 ton per hectare provided an adequate initial growth of coffee plants.
\end{abstract}

Key words - Coffea canephora. Nutrients Source. Nutrients Availability. Growth.

\footnotetext{
* Autor para correspondência

${ }^{1}$ Recebido para publicação em 27/08/2009; aprovado em 29/12/2010

Trabalho financiado com recursos do Banco do Nordeste (BNB)/Fundeci

${ }^{2}$ Pesquisador do Incaper, CRDR-Centro Serrano, Venda Nova do Imigrante-ES, Brasil, guarconi@incaper.es.gov.br

${ }^{3}$ Pesquisador do Incaper, CRDR-Nordeste, Linhares-ES, Brasil, fanton@incaper.es.gov.br
} 


\section{Introdução}

O resíduo de beneficiamento do granito é obtido após o corte dos blocos em teares mecânicos. Nesse processo, é adicionada a cal visando formar uma liga com a limalha de ferro utilizada na abrasão. $O$ resíduo é então descartado em lagoas de decantação ou nos próprios pátios das beneficiadoras de onde é transportado para depósitos industriais. No entanto, a proposta de simples descarte desses resíduos denota completa falta de conhecimento sobre sua composição e sobre formas alternativas de utilização. É importante ressaltar que cada tear, trabalhando em sua capacidade máxima, pode gerar até $5 \mathrm{t} \mathrm{dia}^{-1}$ de resíduos.

Do ponto de vista mineralógico, o granito é constituído basicamente por quartzo, feldspatos, mica e calcita (Menezes et al., 2002). Assim, devido à sua mineralogia, constata-se que o resíduo de beneficiamento do granito possa conter teores significativos de alguns elementos químicos considerados macro e micronutrientes para as plantas. Dentre os macronutrientes, destacam-se potássio $(\mathrm{K})$, cálcio $(\mathrm{Ca})$ e magnésio $(\mathrm{Mg})$. Em relação aos micronutrientes, merece destaque o Fe que está presente no resíduo devido à utilização da limalha. Apesar da presença vantajosa de alguns nutrientes, é também possível que o resíduo de beneficiamento do granito apresente quantidades apreciáveis de $\mathrm{Cd}, \mathrm{Cr}, \mathrm{Ni}$ e $\mathrm{Pb}$ que podem ser tóxicos para as plantas e/ou nocivos à saúde humana quando em concentrações elevadas.

A utilização do resíduo de beneficiamento do granito como fertilizante para diversas culturas tem sido testada. Vasconcelos et al. (2003a, 2003b) e Rosen (2002) observaram efeitos positivos desse resíduo e reportaram sua possível utilização na agricultura como fertilizante. Por outro lado, é imperativo destacar que na grande maioria dos trabalhos que utilizaram resíduos de rochas não houve preocupação em se quantificar a liberação de metais pesados por esses materiais e sua possível absorção pelas plantas.

Por ser o granito uma rocha plutônica, é de se esperar que a liberação dos nutrientes a partir deste material seja lenta, como observado por Bolland e Baker (2000). Entretanto, o café é uma planta que exsuda, na rizosfera, ácidos orgânicos de baixo peso molecular como o ácido cítrico (NOGUEIRA et al., 2001), podendo acelerar a solubilização do pó de granito e, consequentemente, aumentar a disponibilidade dos nutrientes para absorção através das raízes, como reportado por Harley e Gilkes (2000).

A utilização do pó de rochas na agricultura vem sendo muito discutida, porém pouco estudada, tanto que o número de artigos publicados em revistas científicas com corpo editorial é insignificante. Portanto, pode-se presumir que o apelo para utilização desses resíduos na agricultura vem sendo justificado a partir de questões ideológicas, carecendo de maior embasamento científico.

Este estudo teve por objetivo avaliar o efeito do resíduo de beneficiamento do granito sobre as características químicas do solo, a absorção de macro, micronutrientes e metais pesados e o desenvolvimento inicial do café conilon, em casa de vegetação, utilizando-se duas amostras de um mesmo solo e duas doses de calcário.

\section{Material e métodos}

$\mathrm{O}$ experimento foi realizado em condições controladas de casa de vegetação, no Centro Regional de Desenvolvimento Rural Nordeste (CRDR-Nordeste; Linhares - ES; Latitude $19^{\circ} 04^{\prime} \mathrm{S}$ e Longitude $40^{\circ} 05^{\prime} \mathrm{W}$, com Clima Aw pela Classificação de Köppen), pertencente ao Incaper. Foi utilizado resíduo de beneficiamento do granito obtido em lagoa de descarte de uma beneficiadora (teares) de Nova Venécia - ES (TAB. 1).

Os fatores estudados foram: doses do resíduo de beneficiamento do granito, duas doses de calcário dolomítico e duas amostras de um Latossolo VermelhoAmarelo coeso (superficial: 0 a $20 \mathrm{~cm}$ de profundidade (SUP) e subsuperficial: 20 a $40 \mathrm{~cm}$ de profundidade (SUB), (TAB. 2).

Os fatores foram combinados segundo o fatorial $6 \times 2 \times 2$, sendo: seis doses de resíduo $(0,0 ; 1,5 ; 3,0 ; 6,0$; 9,0 e $15 \mathrm{~g} \mathrm{dm}^{-3}$, equivalentes a $0 ; 3 ; 6 ; 12 ; 18$ e $30 \mathrm{t} \mathrm{ha}^{-1}$ ) $\mathrm{x}$ duas doses de calcário dolomítico $(0,4$ e 1,2 vezes a necessidade de calagem, calculada pelo método da

Tabela 1 - Caracterização ${ }^{1 /}$ do resíduo de beneficiamento do granito (RBG) utilizado no experimento

\begin{tabular}{|c|c|c|c|c|c|c|c|c|c|c|c|c|}
\hline Material & $\mathrm{P}$ & $\mathrm{K}$ & $\mathrm{Ca}$ & $\mathrm{Mg}$ & $\mathrm{Zn}$ & $\mathrm{Fe}$ & $\mathrm{Mn}$ & $\mathrm{Cu}$ & $\mathrm{Cd}$ & $\mathrm{Cr}$ & $\mathrm{Pb}$ & $\mathrm{Ni}$ \\
\hline $\mathrm{RBG}$ & 0,051 & 0,34 & 1,62 & 0,22 & 40 & 25.200 & 236 & 20 & 1,6 & 29,6 & 12 & 26,6 \\
\hline
\end{tabular}

${ }^{1 /} \mathrm{HCl}_{2} \mathrm{~mol} / \mathrm{L}$ 
Tabela 2 - Resultados analíticos das amostras de solo superficial (SUP) e subsuperficial (SUB) utilizadas no experimento

\begin{tabular}{ccccccccccccc}
\hline Solo & $\mathrm{pH}^{1 /}$ & $\mathrm{P}^{2 /}$ & $\mathrm{K}^{2 /}$ & $\mathrm{Ca}^{2+3 /}$ & $\mathrm{Mg}^{2+3 /}$ & $\mathrm{Al}^{3+3 /}$ & $\mathrm{MO}^{4 /}$ & $\mathrm{P}-\mathrm{rem}^{5 /}$ & $\mathrm{t}$ & $\mathrm{T}$ & $\mathrm{V}$ & $\mathrm{m}$ \\
\hline & & $-----\mathrm{mg} / \mathrm{dm}^{3}----$ & -------- & $\mathrm{cmol}_{\mathrm{c}} / \mathrm{dm}^{3}-----$ & $\mathrm{dag} / \mathrm{kg}$ & $\mathrm{mg} / \mathrm{L}$ & $\mathrm{cmol}_{\mathrm{c}} / \mathrm{dm}^{3}$ & $-----\%$----- \\
SUP & 5,0 & 2,5 & 15 & 1,40 & 0,30 & 0,10 & 1,85 & 47,24 & 1,84 & 3,74 & 46,5 & 5,4 \\
SUB & 3,9 & 0,7 & 10 & 0,53 & 0,17 & 1,22 & 0,44 & 24,36 & 1,95 & 3,63 & 20,0 & 62,7 \\
\hline
\end{tabular}

${ }^{1 / p H}$ em água (1:2,5); ${ }^{2} \mathrm{P}$ e K disponíveis (Mehlich-1); ${ }^{3 /} \mathrm{Ca}^{2+}, \mathrm{Mg}^{2+} \mathrm{e} \mathrm{Al}{ }^{3+}\left(\mathrm{KCl}, 1 \mathrm{~mol} \mathrm{~L}{ }^{-1}\right),{ }^{4}$ Walkley \& Black; ${ }^{5 /} \mathrm{P}-\mathrm{rem}$ (P em solução após agitação de $60 \mathrm{mg} \mathrm{L}-1$ de $\mathrm{P}$ em $\mathrm{CaCl}_{2} 0,01$ mol L-1, na relação solo:solução de 1:10, por $1 \mathrm{~h}$ (Alvarez V. et al., 2000)

Saturação por Bases, utilizando-se $\mathrm{Ve}=55 \%$ ) $\mathrm{x}$ duas amostras de um mesmo solo, gerando um total de 24 tratamentos. Os tratamentos foram distribuídos em blocos ao acaso com três repetições, perfazendo um total de 72 unidades experimentais, sendo composta, cada uma, por um vaso contendo solo e uma planta de café conilon. Com base no Manual de Recomendação de Calagem e Adubação para o Estado do Espírito Santo (LANI et al., 2007), foram aplicadas, como fatores constantes, uma dose de $100 \mathrm{mg} \mathrm{dm}^{-3} \mathrm{de} \mathrm{P}$, na forma de superfosfato simples (misturado ao solo dos vasos), e uma dose de $250 \mathrm{mg} \mathrm{dm}^{-3}$ de $\mathrm{N}$, na forma de uréia (quatro parcelamentos).

As duas amostras de solo foram passadas por peneira de $6 \mathrm{~mm}$ e separadas em subamostras de $9 \mathrm{dm}^{3}$, com base na densidade (SUP $=1,14 \mathrm{~g} \mathrm{~cm}^{-3}$ e SUB $=1,30 \mathrm{~g} \mathrm{~cm}^{-3}$ ). Os tratamentos foram aplicados misturando-se de forma homogênea as doses de resíduo de granito, de calcário dolomítico e de superfosfato simples com as subamostras de solo. Cada subamostra foi disposta em vaso de $10 \mathrm{~L}$ e incubada por 30 dias com aplicação de água destilada, mantendo-se a umidade das subamostras de solo próxima à capacidade de campo.

Concluído o período de incubação, foram transplantadas três estacas de um mesmo clone de café conilon para o centro dos vasos. Após o pegamento das mudas, foi selecionada a planta de maior vigor, sendo as outras duas eliminadas. As plantas foram cultivadas durante 12 meses, com irrigação periódica realizada com água destilada.

No período que antecedeu a colheita, foi determinada a altura das plantas do coleto ao ápice. Após a colheita, as plantas foram separadas em parte aérea e raízes, as quais foram secas em estufa com ventilação forçada a $70^{\circ} \mathrm{C}$, até peso constante, sendo, posteriormente, determinada a matéria seca da parte aérea e das raízes. A parte aérea das plantas foi então moída em moinho tipo Willey e mineralizada por via úmida com digestão nítricoperclórica, sendo determinados os teores de $\mathrm{P}, \mathrm{K}, \mathrm{Ca}, \mathrm{Mg}$, $\mathrm{Zn}, \mathrm{Cu}, \mathrm{Fe}, \mathrm{Mn}, \mathrm{Cd}, \mathrm{Cr}$, Ni e $\mathrm{Pb}$ na parte aérea, segundo Tedesco et al. (1995). O solo dos vasos foi seco à sombra, peneirado e analisado, determinando-se as seguintes características: $\mathrm{pH}\left(\mathrm{H}_{2} \mathrm{O}\right), \mathrm{Al}^{3+}$, matéria orgânica (MO), $\mathrm{P}, \mathrm{K}, \mathrm{Ca}^{2+}$ e $\mathrm{Mg}^{2+}$, segundo Embrapa (1997), e fósforo remanescente (P-rem), segundo Alvarez V. et al. (2000).

Foram calculados contrastes ortogonais para comparar o efeito do tipo de amostra de solo utilizada (SUP e SUB) e das doses de calcário sobre o resíduo de beneficiamento do granito. Foram ajustados, além disso, modelos de regressão para avaliar os efeitos das doses do resíduo de beneficiamento do granito, nas duas doses de calcário, em cada tipo de amostra de solo utilizada (SUP e SUB).

\section{Resultados e discussão}

A amostra superficial do solo (SUP) apresentou, em média, maior valor de $\mathrm{pH}$, maiores teores de matéria orgânica $(\mathrm{MO})$ e de fósforo remanescente (P-rem) e menor teor de alumínio trocável $\left(\mathrm{Al}^{3+}\right)$ (TAB. 3 e 4$)$. Por apresentar, naturalmente, maior teor de P-rem (TAB. 2), evidenciando uma textura mais arenosa, a (SUP) continha menor número de cargas e consequente menor reatividade, explicando o maior valor de $\mathrm{pH}$ e o menor teor de $\mathrm{Al}^{3+}$, em relação à SUB. Além disso, é também possível que calagens anteriores realizadas na área possam explicar o resultado. Por ser proveniente da camada superficial do solo, onde ocorre deposição de resíduos orgânicos, a (SUP) apresentava, naturalmente, maior teor de $\mathrm{MO}$ do que a amostra subsuperficial do solo (SUB).

$\mathrm{Na}$ (SUB), a elevação da dose de calcário de $(0,4 \times \mathrm{NC})$ para $(1,2 \times \mathrm{NC})$ aumentou seu $\mathrm{pH}$ de forma significativa, em $23 \%$ (TAB. 3 e 4). Efeito inverso foi observado nos teores de $\mathrm{Al}^{3+}$, uma vez que o teor médio foi reduzido em $88 \%$, como seria de se esperar (TAB. 3 e 4). Essa observação é confirmada por Caires et al. (2008), que também observaram aumento no $\mathrm{pH}$ e redução nos teores de $\mathrm{Al}^{3+}$ ao aplicarem doses mais elevadas de calcário. 
Tabelas 3 - Médias de características químicas de duas amostras de um mesmo solo após aplicação de doses de resíduo de beneficiamento do granito e duas doses de calcário

\begin{tabular}{cccccccccc}
\hline Amostra $^{1 /}$ & $\mathrm{NC} \mathrm{x}^{2 /}$ & $\mathrm{pH}$ & $\mathrm{Al}^{3+}$ & $\mathrm{MO}$ & $\mathrm{P}-\mathrm{rem}$ & $\mathrm{P}$ & $\mathrm{K}$ & $\mathrm{Ca}^{2+}$ & $\mathrm{Mg}^{2+}$ \\
\cline { 2 - 9 } & & & $\mathrm{cmol}_{\mathrm{c}} \mathrm{dm}^{-3}$ & $\mathrm{dag} \mathrm{kg}^{-1}$ & $\mathrm{mg} \mathrm{L}$ & $-------\mathrm{mg} \mathrm{dm}^{-3}$------ & $------\mathrm{cmol}_{\mathrm{c}} \mathrm{dm}^{-3}$------ \\
\cline { 2 - 9 } SUP & 0,4 & 4,6 & 0,17 & 1,77 & 54,0 & 34,5 & 20 & 1,58 & 0,13 \\
SUP & 1,2 & 4,8 & 0,13 & 1,76 & 55,2 & 32,8 & 14 & 1,69 & 0,13 \\
SUB & 0,4 & 3,9 & 0,66 & 0,02 & 33,2 & 27,4 & 13 & 1,20 & 0,20 \\
SUB & 1,2 & 4,8 & 0,08 & 0,01 & 34,1 & 25,7 & 21 & 1,64 & 0,45 \\
\hline
\end{tabular}

${ }^{1 / A m o s t r a s ~ s u p e r f i c i a l ~(S U P) ~ e ~ s u b s u p e r f i c i a l ~(S U B) ~ d e ~ u m ~ m e s m o ~ s o l o ; ~}{ }^{2}$ Dose de calcário: $0,4 \mathrm{x}$ a necessidade de calagem e $1,2 \mathrm{x}$ a necessidade de calagem

Tabela 4 - Valores de F calculados para contrastes de características químicas de duas amostras de um mesmo solo após aplicação de doses de resíduo de beneficiamento do granito e duas doses de calcário

\begin{tabular}{llllrrrrc}
\hline \multicolumn{1}{c}{ Contrastes } & $\mathrm{pH}$ & $\mathrm{Al}^{3+}$ & $\mathrm{MO}$ & $\mathrm{P}-\mathrm{rem}$ & $\mathrm{P}$ & $\mathrm{K}$ & $\mathrm{Ca}^{2+}$ & $\mathrm{Mg}^{2+}$ \\
\hline $\mathrm{SUP}_{\mathrm{vs} \mathrm{SUB}}{ }^{1 /}$ & $0,195^{* *}$ & $0,094^{* *}$ & $6,764^{* *}$ & $921,58^{* *}$ & $115,6^{* *}$ & $1,8^{\mathrm{ns}}$ & $0,101^{*}$ & $0,086^{* *}$ \\
$0,4 \mathrm{NC}$ vs $1,2 \mathrm{NC} d / \mathrm{SUP}^{2 /}$ & $0,034^{\mathrm{ns}}$ & $0,002^{\mathrm{ns}}$ & $0,000^{\mathrm{ns}}$ & $1,28^{\mathrm{ns}}$ & $3,1^{\mathrm{ns}}$ & $26,7^{\mathrm{o}}$ & $0,012^{\mathrm{ns}}$ & $0,000^{\mathrm{ns}}$ \\
$0,4 \mathrm{NC}$ vs $1,2 \mathrm{NC} d / \mathrm{SUB}^{2 /}$ & $0,780^{* *}$ & $0,338^{* *}$ & $0,000^{\mathrm{ns}}$ & $0,84^{\mathrm{ns}}$ & $3,0^{\mathrm{ns}}$ & $69,4^{* *}$ & $0,188^{* *}$ & $0,063^{* *}$ \\
\hline
\end{tabular}

${ }^{1 / A m o s t r a}$ superficial vs Amostra subsuperficial do solo; ${ }^{2 / 0,4}$ vezes a necessidade de calagem vs 1,2 vezes a necessidade de calagem, na amostra superficial e na amostra subsuperficial; ${ }^{* *},{ }^{*},{ }^{\circ} \mathrm{e}^{\mathrm{ns}}$ significativo a $1,5,10 \%$ de probabilidade e não significativo, respectivamente

Não foi observado efeito da calagem nas características de acidez da amostra superficial do solo (SUP) (TAB. 3 e 4), uma vez que a dose básica calculada pelo método da Saturação por Bases foi muito reduzida nessa situação $(0,32$ t/ha de calcário). Guarçoni $M$. et al. (2007), avaliando diferentes métodos de cálculo da Necessidade de Calagem (NC) para 380 amostras de solos de $\mathrm{MG}$, demonstraram que o cálculo da NC pelo método da Saturação por Bases pode gerar doses extremamente reduzidas, caso a CTC pH 7 do solo seja baixa, mesmo que a saturação por bases (V) esteja abaixo daquela adequada para a cultura do café, como foi o caso da SUP no presente estudo (TAB. 2). É interessante ressaltar que a dose básica de calagem calculada para a SUB (1,27 t/ha de calcário) foi quatro vezes maior do que a dose calculada para a SUP (0,32 t/ha de calcário), ainda que os valores de CTC $\mathrm{pH}$ 7,0 (T) das duas amostras tenham sido bem próximos (TAB. 2). Isso ocorreu porque a saturação por bases na SUB era extremamente reduzida (TAB. 2).

Com aplicação de doses crescentes do resíduo de beneficiamento do granito, os valores de $\mathrm{pH}$ de ambas as amostras do solo (SUP e SUB) aumentaram linearmente, sendo os teores de $\mathrm{Al}^{3+}$ reduzidos da mesma forma (TAB. 5). Entretanto, como ficou evidenciado pelos reduzidos valores dos coeficientes das equações, a declividade dos modelos lineares selecionados para essas situações foi pequena (TAB. 5), demonstrando baixa eficiência do resíduo de beneficiamento do granito na correção do solo. Nesse caso, para ambas as amostras do solo, a aplicação de $10 \mathrm{t} \mathrm{ha}^{-1}$ de resíduo iria aumentar o pH em apenas três décimos, aproximadamente.

A SUP apresentou maiores teores de $\mathrm{P}$ e $\mathrm{Ca}^{2+} \mathrm{e}$ menor de $\mathrm{Mg}^{2+}$ em relação à SUB (TAB. 3 e 4). A amostra superficial do solo (SUP) já apresentava maior teor de $\mathrm{Ca}^{2+}$ do que a SUB (TAB. 2). Para o $\mathrm{Mg}^{2+}$, uma maior lixiviação e/ou absorção pelas plantas cultivadas na SUP pode ter reduzido os teores em relação à SUB. Entretanto, para o caso do P, o extrator Mehlich 1, utilizado na análise, apresenta maior capacidade de recuperação de $\mathrm{P}$ em solo com menor capacidade tampão (Rheinheimer et al., 2003), como é o caso da SUP, explicando o maior teor nesta amostra após a adubação inicial com superfosfato simples.

Por sua vez, os teores de K não foram diferentes entre as duas amostras do mesmo solo (TAB. 3 e 4), uma vez que as doses de resíduo foram equivalentes em ambas e a recuperação de K, pelo extrator Mehlich 1, sofre pouco efeito da capacidade tampão do solo, como observado por Villa et al. (2004).

$\mathrm{O}$ aumento da dose de calcário na SUB elevou a

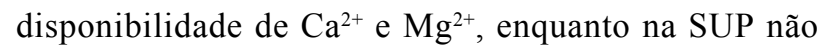
foi observado qualquer efeito sobre os teores médios de $\mathrm{Ca}^{2+}$ e $\mathrm{Mg}^{2+}$ (TAB. 3 e 4). Esse fato ocorreu porque as doses de calcário calculadas para a amostra superficial 
(SUP) foram muito baixas $\left(0,4 \times \mathrm{NC}=0,127 \mathrm{t} \mathrm{ha}^{-1} \mathrm{e}\right.$ $\left.1,2 \times \mathrm{NC}=0,382 \mathrm{tha}^{-1}\right)$, reduzindo a possibilidade de diferença estatística.

Considerando os macronutrientes analisados, apenas $\mathrm{K} \mathrm{e} \mathrm{Ca}^{2+}$ sofreram influência de doses crescentes

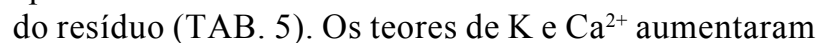
de forma linear nas duas amostras do solo (SUP e SUB), considerando as duas doses de calcário, exceção feita ao teor de $\mathrm{K}$, que sofreu efeito quadrático na SUP na menor dose de calcário e na SUB na maior dose de calcário (TAB. 5). Nos dois casos, as doses de resíduo de beneficiamento do granito que proporcionaram os maiores teores de $\mathrm{K}$, sendo estes equivalentes nas duas amostras, foram $28,5 \mathrm{t} \mathrm{ha}^{-1}$ de resíduo na SUP e $18,7 \mathrm{t} \mathrm{ha}^{-1}$ de resíduo na SUB. Assim, fica claro que a aplicação do resíduo de beneficiamento do granito é muito mais eficiente, quanto à disponibilização de $\mathrm{K}$, em solo com maior capacidade tampão e maior reação, caso da amostra subsuperficial (SUB), que apresentou menor teor de P-rem (TAB. 2, 3 e 4). Esse fato é explicado por Harley e Gilkes (2000), para quem solos mais reativos, que reduzem o teor de $\mathrm{K}$ na solução do solo por meio de reações de adsorção, provocam maior dissolução de resíduos de rocha em relação a solos menos reativos.

Foram observados, na amostra subsuperficial do solo (SUB), maiores teores de $\mathrm{K}$ na maior dose de calcário, enquanto na amostra superficial do solo (SUP), maiores teores de $\mathrm{K}$ foram observados na menor dose de calcário (TAB. 3, 4 e 5). Esse fato demonstra que, o pH de qualquer solo adubado com o resíduo de beneficiamento do granito deve ser baixo o suficiente para que ocorra a solubilização do mesmo, como reportado por Harley e Gilkes (2000), mas, em contrapartida, deve ser alto o suficiente para que não ocorra lixiviação excessiva do K liberado pelo resíduo. Em ambas as amostras estudadas (SUP e SUB), ao se transportar as doses de resíduo que proporcionaram os maiores teores de $\mathrm{K}$ nas duas situações, para as relativas equações de pH (TAB. 5), observaram-se valores de $\mathrm{pH}$ correspondentes a 5,0.

Tabela 5 - Equações de regressão de características e de teores de macronutrientes em duas amostras de um mesmo solo (SUP e SUB ${ }^{1 /}$ ), após aplicação de doses de resíduo de beneficiamento do granito e duas doses de calcário (0,4 ou 1,2 NC)

\begin{tabular}{|c|c|c|}
\hline Variável dependente & Equação ${ }^{2 /}$ & $\mathrm{R}^{2}$ \\
\hline \multicolumn{3}{|c|}{0,4 NC SUP } \\
\hline $\mathrm{pH}$ & $\hat{Y}=4,31+0,0241 * * \mathrm{X}$ & 0,93 \\
\hline $\mathrm{Al}^{3+}$ & $\hat{Y}=0,237-0,0062^{* *} \mathrm{X}$ & 0,92 \\
\hline $\mathrm{K}$ & $\hat{Y}=12,84+0,9049 \mathrm{X}-0,0159 * \mathrm{X}^{2}$ & 0,88 \\
\hline $\mathrm{Ca}^{2+}$ & $\hat{Y}=1,28+0,0260 * * \mathrm{X}$ & 0,99 \\
\hline \multicolumn{3}{|c|}{1,2 NC SUP } \\
\hline $\mathrm{pH}$ & $\hat{Y}=4,43+0,0294 * * \mathrm{X}$ & 0,97 \\
\hline $\mathrm{Al}^{3+}$ & $\hat{Y}=0,175-0,0043 * * \mathrm{X}$ & 0,93 \\
\hline $\mathrm{K}$ & $\hat{Y}=11,63+0,2583 * * \mathrm{X}$ & 0,78 \\
\hline $\mathrm{Ca}^{2+}$ & $\hat{Y}=1,47+0,0190 * * \mathrm{X}$ & 0,99 \\
\hline \multicolumn{3}{|c|}{0,4 NC SUB } \\
\hline $\mathrm{pH}$ & $\hat{Y}=3,63+0,0263 * * \mathrm{X}$ & 0,99 \\
\hline $\mathrm{Al}^{3+}$ & $\hat{Y}=0,960-0,0264 * * \mathrm{X}$ & 0,95 \\
\hline $\mathrm{K}$ & $\hat{Y}=7,67+0,4342 * * \mathrm{X}$ & 0,90 \\
\hline $\mathrm{Ca}^{2+}$ & $\hat{Y}=1,01+0,0168^{* *} \mathrm{X}$ & 0,91 \\
\hline \multicolumn{3}{|c|}{1,2 NC SUB } \\
\hline $\mathrm{pH}$ & $\hat{Y}=4,50+0,0277^{* *} \mathrm{X}$ & 0,87 \\
\hline $\mathrm{Al}^{3+}$ & $\hat{Y}=0,129-0,0046^{* *} \mathrm{X}$ & 0,71 \\
\hline $\mathrm{K}$ & $\hat{Y}=9,19+2,26 \mathrm{X}-0,0604 * * \mathrm{X}^{2}$ & 0,89 \\
\hline $\mathrm{Ca}^{2+}$ & $\hat{Y}=1,48+0,0134 * * \mathrm{X}$ & 0,78 \\
\hline
\end{tabular}

${ }^{1 / A}$ Amostra superficial (SUP) e Amostra subsuperficial (SUB) do solo; ${ }^{2 /}$ Equações não apresentadas representam $\hat{Y}=\bar{Y} ;{ }^{* *},{ }^{*} \mathrm{e}{ }^{0}$ Significativo aos níveis de $1 ; 5$ e $10 \%$ de probabilidade, respectivamente 
A maior absorção de $\mathrm{Zn}, \mathrm{Cu}$ e $\mathrm{Mn}$ pelas plantas cultivadas na SUP em relação às cultivadas na SUB (TAB. 6 e 7) foi causada pelo maior teor de matéria orgânica presente na primeira (TAB. 2; 3 e 4), uma vez que esta foi coletada na camada superficial do terreno, onde há maior acúmulo de matéria orgânica e consequentemente maior disponibilização de micronutrientes quando ocorre a mineralização. Esse resultado é corroborado por Oliveira et al. (2004), que observaram maiores teores de nutrientes em solos com maior teor de matéria orgânica.

Quando a dose de calcário passou de $0,4 \times \mathrm{NC}$ para 1,2 x NC, foi observada menor absorção de $\mathrm{Fe}$ pelas plantas cultivadas na SUB e de Mn e Fe pelas cultivadas na SUP (TAB. 6 e 7). A menor absorção desses dois micronutrientes metálicos é decorrente da redução de sua disponibilidade no solo, em conseqüência da elevação do $\mathrm{pH}$ causada pelo maior nível de calagem, como observado para $\mathrm{Mn}$ e $\mathrm{Zn}$ por Nachtigall et al. (2009).

O teor de Mn na parte aérea das plantas foi o que sofreu maior influência das doses de resíduo de beneficiamento do granito, sendo que sua absorção foi reduzida linearmente na SUP, para as duas doses de calcário aplicadas (TAB. 8). Já na SUB, os teores de Mn na parte aérea das plantas sofreram efeito quadrático, na dose de $0,4 \times \mathrm{NC}$, e raiz quadrático, na dose de 1,2 x NC (TAB. 8).

Os teores de $\mathrm{Zn}$ na parte aérea foram menos afetados pelas doses de resíduo de beneficiamento do granito do que os teores de $\mathrm{Mn}$, como comprovam as declividades das curvas selecionadas (TAB. 8). Os teores dos demais micronutrientes estudados não sofreram efeito consistente das doses de resíduo do beneficiamento do granito (TAB. 8).

A partir desses resultados, fica claro que a absorção de micronutrientes pelas plantas de café conilon foi mais afetada pela elevação do $\mathrm{pH}$ do solo, o qual sofreu influência da aplicação do resíduo de beneficiamento do granito, do que pelos conteúdos de micronutrientes presentes neste material. Assim, o resíduo de beneficiamento do granito não deve ser considerado como fonte de micronutrientes para o crescimento inicial do café.

Tabela 6 - Médias dos teores de micronutrientes e metais pesados na parte aérea de plantas de café conilon cultivadas em duas amostras de um mesmo solo, após aplicação de doses de resíduo de beneficiamento do granito e duas doses de calcário

\begin{tabular}{|c|c|c|c|c|c|c|c|c|c|}
\hline \multirow{2}{*}{ Amostra $^{1}$} & \multirow{2}{*}{$\mathrm{NC} x^{2 /}$} & $\mathrm{Zn}$ & $\mathrm{Cu}$ & $\mathrm{Mn}$ & $\mathrm{Fe}$ & $\mathrm{Cd}$ & $\mathrm{Cr}$ & $\mathrm{Ni}$ & $\mathrm{Pb}$ \\
\hline & & \multicolumn{8}{|c|}{ - } \\
\hline SUP & 0,4 & 10,6 & 9,0 & 118 & 145 & 1,11 & 0,14 & 2,42 & 1,61 \\
\hline SUP & 1,2 & 10,2 & 9,2 & 84 & 114 & 1,95 & 0,12 & 2,61 & 1,89 \\
\hline SUB & 0,4 & 9,3 & 7,2 & 53 & 147 & 0,63 & 0,25 & 3,00 & 0,65 \\
\hline SUB & 1,2 & 8,9 & 8,8 & 39 & 115 & 0,95 & 0,17 & 1,95 & 1,57 \\
\hline
\end{tabular}

${ }^{1 / A m o s t r a s ~ s u p e r f i c i a l ~(S U P) ~ e ~ s u b s u p e r f i c i a l ~(S U B) ~ d e ~ u m ~ m e s m o ~ s o l o ; ~}{ }^{2}$ Dose de calcário: $0,4 \mathrm{x}$ a necessidade de calagem e $1,2 \mathrm{x}$ a necessidade de calagem

Tabela 7 - Valores de F calculado para contrastes de teores de micronutrientes e de metais pesados na parte aérea de plantas de café conilon cultivadas em duas amostras de um mesmo solo, após aplicação de doses de resíduo de beneficiamento do granito e duas doses de calcário

\begin{tabular}{lcccccccc}
\hline \multicolumn{1}{c}{ Contrastes } & $\mathrm{Zn}$ & $\mathrm{Cu}$ & $\mathrm{Mn}$ & $\mathrm{Fe}$ & $\mathrm{Cd}$ & $\mathrm{Cr}$ & $\mathrm{Ni}$ & $\mathrm{Pb}$ \\
\hline SUP vs SUB $^{1 /}$ & $4,062^{*}$ & $2,59^{\circ}$ & $7522,5^{* *}$ & $36,0^{\text {ns }}$ & $1,257^{* *}$ & $0,016^{\text {ns }}$ & $0,002^{\text {ns }}$ & $0,780^{\text {ns }}$ \\
$0,4 \mathrm{NC}$ vs $1,2 \mathrm{NC} d / \mathrm{SUP}^{2 /}$ & $0,147^{\text {ns }}$ & $0,02^{\text {ns }}$ & $1201,8^{* *}$ & $920,1^{*}$ & $0,694^{* *}$ & $0,001^{\text {ns }}$ & $0,040^{\text {ns }}$ & $0,063^{\text {ns }}$ \\
$0,4 \mathrm{NC}$ vs $1,2 \mathrm{NC} d / \mathrm{SUB}^{2 /}$ & $0,203^{\text {ns }}$ & $2,61^{\circ}$ & $200,7^{\text {ns }}$ & $1.067,1^{*}$ & $0,123^{\circ}$ & $0,007^{\text {ns }}$ & $1,103^{\text {ns }}$ & $0,780^{\text {ns }}$ \\
\hline
\end{tabular}

${ }^{1 / A m o s t r a}$ superficial vs Amostra subsuperficial do solo; ${ }^{2 /} 0,4$ vezes a necessidade de calagem vs 1,2 vezes a necessidade de calagem, na amostra superficial e na amostra subsuperficial; ${ }^{* *},{ }^{*},{ }^{\circ} \mathrm{e}^{\mathrm{ns}}$ significativo a $1,5,10 \%$ de probabilidade e não significativo, respectivamente 
Tabela 8 - Equações de regressão de teores de micronutrientes e de metais pesados na parte aérea de plantas de café conilon cultivadas em duas amostras de um mesmo solo (SUP e $\mathrm{SUB}^{1 /}$ ), após aplicação de doses de resíduo de beneficiamento do granito e duas doses de calcário $(0,4$ e $1,2 \mathrm{NC})$

\begin{tabular}{|c|c|c|}
\hline Variável dependente & Equação $^{2 /}$ & $\mathrm{R}^{2}$ \\
\hline \multicolumn{3}{|c|}{0,4 NC SUP } \\
\hline $\mathrm{Mn}$ & $\hat{Y}=147,84-2,58 * * X$ & 0,97 \\
\hline $\mathrm{Cd}$ & $\hat{Y}=0,876+0,0209 * * \mathrm{X}$ & 0,66 \\
\hline \multicolumn{3}{|c|}{1,2 NC SUP } \\
\hline $\mathrm{Mn}$ & $\hat{Y}=111,32-2,42 * * \mathrm{X}$ & 0,74 \\
\hline $\mathrm{Zn}$ & $\hat{Y}=11,09-0,0755^{* *} \mathrm{X}$ & 0,93 \\
\hline $\mathrm{Cd}$ & $\hat{Y}=1,72+0,1655^{*} \sqrt{ } \mathrm{X}-0,0218 \mathrm{X}$ & 0,91 \\
\hline \multicolumn{3}{|c|}{0,4 NC SUB } \\
\hline $\mathrm{Mn}$ & $\hat{Y}=38,23+2,70 \mathrm{X}-0,0703 * * \mathrm{X}^{2}$ & 0,97 \\
\hline $\mathrm{Zn}$ & $\hat{Y}=8,07+0,2249 \mathrm{X}-0,0057^{*} \mathrm{X}^{2}$ & 0,72 \\
\hline $\mathrm{Cd}$ & $\hat{Y}=0,465+0,0132 * * \mathrm{X}$ & 0,84 \\
\hline \multicolumn{3}{|c|}{1,2 NC SUB } \\
\hline $\mathrm{Mn}$ & $\hat{Y}=56,41-14,15^{* *} \sqrt{\mathrm{X}+2,00 \mathrm{X}}$ & 0,97 \\
\hline
\end{tabular}

${ }^{1 / A m o s t r a}$ superficial (SUP) e Amostra subsuperficial (SUB) do solo; ${ }^{2 /}$ Equações não apresentadas representam $\hat{Y}=\bar{Y} ; * *, *$ e 0 Significativo aos níveis de 1,5 e $10 \%$ de probabilidade, respectivamente

Reduzido efeito da aplicação do resíduo de beneficiamento do granito foi observado sobre os teores de metais pesados na parte aérea das plantas de café conilon (TAB. 6; 7 e 8).

Apenas os teores de $\mathrm{Cd}$ apresentaram diferenças mais marcantes, especialmente comparando-se as quantidades absorvidas pelas plantas cultivadas na SUP e na SUB, e quando se elevou a dose de calcário de 0,4 x NC para 1,2 x NC na SUP (TAB. 6 e 7). Entretanto, essas diferenças foram muito mais influenciadas pelo maior teor de matéria orgânica da amostra superficial (SUP) (TAB. 2) e por possíveis contaminações oriundas do calcário, do que pelo resíduo de beneficiamento do granito, uma vez que os grupos comparados por meio dos contrastes receberam as mesmas doses de resíduo. Essa inferência é reforçada por Amaral Sobrinho et al. (1992), que reportam ser o $\mathrm{Cd}$ o metal pesado mais frequentemente encontrado em vários corretivos de solo avaliados.

A aplicação de doses crescentes de resíduo de beneficiamento do granito casou efeito linear positivo na absorção de $\mathrm{Cd}$ pelas plantas de café conilon, quando a dose de calcário foi baixa, nas duas amostras de solo estudadas (TAB. 8). Entretanto, quando se elevou o $\mathrm{pH}$ do solo $(1,2 \times \mathrm{NC})$, não se observou efeito do resíduo na absorção de $\mathrm{Cd}$, ou os teores apresentaram tendência de redução a partir de uma dose de $14,4 \mathrm{t} \mathrm{ha}^{-1}$ de resíduo (TAB. 8), o que, no caso, correspondeu a um pH de 4,9.
Nesse sentido, Amaral Sobrinho et al. (1997) observaram que valores mais elevados $\mathrm{pH}$ favoreceram a passagem dos metais pesados de formas mais solúveis para formas de menor solubilidade.

De qualquer forma, os teores de metais pesados na parte aérea do café conilon ficaram abaixo dos limites críticos estabelecidos por Alloway (1995) para a parte aérea das plantas, sendo esses de 5 a 30 para $\mathrm{Cd}$ e $\mathrm{Cr}, 10$ a 100 para $\mathrm{Ni}$ e 30 a $300 \mathrm{mg} \mathrm{kg}^{-1}$ para $\mathrm{Pb}$. Ainda assim, se a parte aérea como um todo apresentasse teor mais elevado de algum metal pesado, isso não seria, a princípio, um problema para plantas de café, uma vez que Berton (2000) relata que os grãos contêm menor concentração de metais pesados do que a parte vegetativa do cafeeiro.

Os teores de macronutrientes determinados na parte aérea do cafeeiro conilon, no presente estudo, não podem ser comparados a valores tabelados para a cultura, uma vez que estes são determinados apenas em um grupo de folhas, o que não apresenta correspondência com os teores determinados em toda a parte aérea. Portanto, mesmo que os teores de macronutrientes apresentados na Tabela 9 pareçam baixos, nada pode ser afirmado em relação aos valores tabelados para a cultura. 
Tabela 9 - Médias dos teores de macronutrientes na parte aérea, altura (ALT), matéria seca de raízes (MSR) e matéria seca da parte aérea (MSPA) de plantas de café conilon cultivadas em duas amostras de um mesmo solo, após aplicação de doses de resíduo de beneficiamento do granito e duas doses de calcário

\begin{tabular}{|c|c|c|c|c|c|c|c|c|}
\hline \multirow{2}{*}{ Amostra $^{1 /}$} & \multirow{2}{*}{$\mathrm{NC} \mathrm{x}^{2 /}$} & $\mathrm{P}$ & $\mathrm{K}$ & $\mathrm{Ca}$ & $\mathrm{Mg}$ & \multirow{2}{*}{$\frac{\mathrm{ALT}}{\mathrm{cm}}$} & \multirow{2}{*}{\multicolumn{2}{|c|}{ MSR $\quad$ MSPA }} \\
\hline & & \multicolumn{4}{|c|}{--------------------- $\mathrm{mg} \mathrm{kg}^{-1}$-------------------- } & & & \\
\hline SUP & 0,4 & 0,11 & 1,11 & 1,05 & 0,25 & 38,3 & 15,1 & 39,7 \\
\hline SUP & 1,2 & 0,11 & 1,08 & 0,99 & 0,31 & 38,8 & 16,8 & 41,5 \\
\hline SUB & 0,4 & 0,09 & 1,51 & 0,81 & 0,18 & 32,5 & 4,1 & 15,8 \\
\hline SUB & 1,2 & 0,09 & 1,62 & 0,88 & 0,26 & 25,9 & 2,3 & 10,2 \\
\hline
\end{tabular}

${ }^{1 / A m o s t r a s ~ s u p e r f i c i a l ~(S U P) ~ e ~ s u b s u p e r f i c i a l ~(S U B) ~ d e ~ u m ~ m e s m o ~ s o l o ; ~}{ }^{2 /}$ Dose de calcário: $0,4 \mathrm{x}$ a necessidade de calagem e $1,2 \mathrm{x}$ a necessidade de calagem

As plantas cultivadas na amostra superficial do solo (SUP) absorveram maiores quantidades de $\mathrm{P}, \mathrm{Ca}$ e $\mathrm{Mg}$ do que as cultivadas na amostra subsuperficial do solo (SUB) (TAB. 9 e 10). Por apresentar menor capacidade de adsorção de $\mathrm{P}$ (TAB. 2), a amostra superficial (SUP) do solo disponibilizou maior quantidade de $\mathrm{P}$ para as plantas, em menor período de tempo, mesmo que as doses de fertilizante fosfatado aplicadas tenham sido iguais para ambas as amostras de solo, como explicado por (RHEINHEIMER et al., 2003). Como a SUP foi coletada na camada superficial do solo, já apresentava teores mais elevados de Ca e Mg do que a SUB (TAB. 2), explicando o resultado.

As plantas cultivadas na SUB, por outro lado, apresentaram maior teor de $\mathrm{K}$ na parte aérea, em relação às cultivadas na SUP (TAB. 9 e 10). A amostra subsuperficial (SUB) apresentava maior CTC efetiva (TAB. 2), o que pode ter reduzido a lixiviação desse nutriente, facilitando a absorção pelas plantas. Além disso, o resíduo de beneficiamento do granito libera mais $\mathrm{K}$ em solo mais reativo, como conseqüência de maior solubilização (HARLEY; GILKES, 2000).

Apenas o teor de $\mathrm{Mg}$ na parte aérea apresentou acréscimo quando se elevou a dose de calcário, para as plantas de café conilon cultivadas em ambas as amostras do solo (TAB. 9 e 10). Nesse caso, pouca influência da calagem foi observada na disponibilização de $\mathrm{P}, \mathrm{K}$ e $\mathrm{Ca}$, tendo em vista que foram aplicadas doses elevadas de $\mathrm{P}$ e o resíduo foi utilizado como fonte primária de $\mathrm{K}$ e Ca. Apenas Mg foi aplicado exclusivamente na forma de calcário, em razão da sua baixa concentração no resíduo de beneficiamento do granito (TAB. 1).

A absorção de K pelas plantas de café conilon sofreu efeito quadrático das doses de resíduo de beneficiamento do granito, para a SUB, em ambas as doses de calcário estudadas e para a SUP na dose de 1,2 x NC, sendo linear positiva para a SUP na menor dose de calcário (TAB. 11). No caso das equações quadráticas, as doses máximas de resíduo de beneficiamento do granito foram 21; 22 e $24 \mathrm{t} \mathrm{ha}^{-1}$, respectivamente. Esses valores correspondem a 672; $704 \mathrm{e}$ $768 \mathrm{~g} \mathrm{cova}^{-1}(40 \times 40 \times 40 \mathrm{~cm})$ de resíduo de beneficiamento do granito, respectivamente.

As doses de resíduo de beneficiamento do granito apresentaram efeito linear positivo sobre a absorção de cálcio na SUB, quando se utilizou a menor dose de calcário (TAB. 11). Já na SUP, foi selecionado o modelo raiz quadrada para a absorção de cálcio, quando se utilizou a maior dose de calcário (TAB. 11). Esses

Tabela 10 - Valores de F calculado para contrastes dos teores de macronutrientes na parte aérea, altura (ALT), matéria seca de raízes (MSR) e matéria seca da parte aérea (MSPA) de plantas de café conilon decorrentes de doses de resíduo de beneficiamento do granito, em dois solos, com duas doses de calcário

\begin{tabular}{|c|c|c|c|c|c|c|c|}
\hline Contrastes & $\mathrm{P}$ & $\mathrm{K}$ & $\mathrm{Ca}$ & $\mathrm{Mg}$ & ALT & MSR & MSPA \\
\hline SUP vs SUB $^{1 /}$ & $0,0006^{*}$ & $0,419 * *$ & $0,059 *$ & $0,006 * *$ & $177,00 * *$ & $340,80 * *$ & $1.524,19 * *$ \\
\hline $0,4 \mathrm{NC}$ vs $1,2 \mathrm{NC} \mathrm{d} / \mathrm{SUP}^{2 /}$ & $0,0000^{\mathrm{ns}}$ & $0,001^{\mathrm{ns}}$ & $0,003^{\mathrm{ns}}$ & $0,003 *$ & $0,23^{\mathrm{ns}}$ & $2,81^{\mathrm{ns}}$ & $3,00^{\mathrm{ns}}$ \\
\hline $0,4 \mathrm{NC}$ vs $1,2 \mathrm{NC} \mathrm{d} / \mathrm{SUB}^{2 /}$ & $0,0000^{\mathrm{ns}}$ & $0,012^{\text {ns }}$ & $0,004^{\mathrm{ns}}$ & $0,006 * *$ & $44,67^{\circ}$ & $3,00^{\mathrm{ns}}$ & $31,55^{\mathrm{ns}}$ \\
\hline
\end{tabular}

${ }^{1 / A m o s t r a}$ superficial vs Amostra subsuperficial do solo; ${ }^{2 /} 0,4$ vezes a necessidade de calagem vs 1,2 vezes a necessidade de calagem, na amostra superficial e na amostra subsuperficial; ${ }^{* *},{ }^{*},{ }^{\circ} \mathrm{e}^{\mathrm{ns}}$ significativo a $1 ; 5 ; 10 \%$ de probabilidade e não significativo, respectivamente 
Tabela 11 - Equações de regressão de teores de macronutrientes na parte aérea e de altura (ALT), matéria seca de raízes (MSR) e matéria seca da parte aérea (MSPA) de plantas de café conilon cultivadas em duas amostras de um mesmo solo (SUP e SUB ${ }^{1 /}$ ), após aplicação de doses de resíduo de beneficiamento do granito e duas doses de calcário $(0,4$ e 1,2 NC)

\begin{tabular}{|c|c|c|}
\hline Variável dependente & Equação $^{2 /}$ & $\mathrm{R}^{2}$ \\
\hline \multicolumn{3}{|c|}{0,4 NC SUP } \\
\hline $\mathrm{K}$ & $\hat{Y}=0,780+0,0287 * * \mathrm{X}$ & 0,80 \\
\hline ALT & $\hat{Y}=34,66+0,739 \mathrm{X}-0,0210 * \mathrm{X}^{2}$ & 0,99 \\
\hline MSR & $\hat{Y}=12,44+0,233^{* *} \mathrm{X}$ & 0,75 \\
\hline MSPA & $\hat{Y}=28,72+1,98 \mathrm{X}-0,050 * * \mathrm{X}^{2}$ & 0,67 \\
\hline \multicolumn{3}{|c|}{1,2 NC SUP } \\
\hline K & $\hat{Y}=0,707+0,057 \mathrm{X}-0,0012^{* *} \mathrm{X}^{2}$ & 0,85 \\
\hline $\mathrm{Ca}$ & $\hat{Y}=0,865+0,1130 * \sqrt{ } \mathrm{X}-0,0151 \mathrm{X}$ & 0,81 \\
\hline MSPA & $\hat{Y}=49,52-0,702 * * \mathrm{X}$ & 0,89 \\
\hline \multicolumn{3}{|c|}{0,4 NC SUB } \\
\hline $\bar{K}$ & $\hat{Y}=1,01+0,0810 \mathrm{X}-0,0019^{* *} \mathrm{X}^{2}$ & 0,86 \\
\hline $\mathrm{Ca}$ & $\hat{Y}=0,652+0,0141^{* * \mathrm{X}}$ & 0,96 \\
\hline ALT & $\hat{Y}=31,28+2,97^{\circ} \sqrt{ } \mathrm{X}-0,637 \mathrm{X}$ & 0,72 \\
\hline MSR & $\hat{Y}=4,95-0,0770 * * \mathrm{X}$ & 0,70 \\
\hline MSPA & $\hat{Y}=11,50+12,40^{* *} \sqrt{ } \mathrm{X}-4,60 \mathrm{X}+0,426^{\circ} \mathrm{X}^{1,5}$ & 0,77 \\
\hline \multicolumn{3}{|c|}{1,2 NC SUB } \\
\hline $\bar{K}$ & $\hat{Y}=1,36+0,040 \mathrm{X}-0,0009 * \mathrm{X}^{2}$ & 0,84 \\
\hline ALT & $\hat{Y}=14,77+6,53^{* *} \sqrt{ } \mathrm{X}-0,680 \mathrm{X}$ & 0,98 \\
\hline MSR & $\hat{Y}=0,676+0,4053 \mathrm{X}-0,0114 * * \mathrm{X}^{2}$ & 0,89 \\
\hline MSPA & $\hat{Y}=3,61+4,26^{*} \sqrt{ } \mathrm{X}-0,500 \mathrm{X}$ & 0,68 \\
\hline
\end{tabular}

${ }^{1 / A m o s t r a ~ s u p e r f i c i a l ~(S U P) ~ e ~ A m o s t r a ~ s u b s u p e r f i c i a l ~(S U B) ~ d o ~ s o l o ; ~}{ }^{2}$ Equações não apresentadas representam $\hat{Y}=\bar{Y} ;{ }^{* *},{ }^{*}{ }^{0}$ Significativo aos níveis de $1 ; 5$ e $10 \%$ de probabilidade, respectivamente

resultados já seriam esperados, uma vez que o resíduo de beneficiamento do granito foi utilizado como fonte de $\mathrm{K}$ e Ca no presente estudo.

A amostra superficial do solo (SUP) proporcionou maior desenvolvimento das plantas de café conilon do que a amostra subsuperficial (SUB), tanto para altura de plantas quanto para matéria seca de raízes e matéria seca da parte aérea (TAB. 9 e 10), pois apresentava maior teor de nutrientes e de matéria orgânica já no início do experimento (TAB. 2).

É também notório que solos mais arenosos, como era o caso da (SUP), quando bem adubados e providos de umidade, podem promover maior crescimento das plantas, uma vez que apresentam menor reação e, conseqüentemente, tendem a disponibilizar mais facilmente os nutrientes contidos nos diversos tipos de fertilizantes solúveis. Além disso, devido ao fato da amostra subsuperficial (SUB) consistir de um
Horizonte B proveniente de tabuleiros costeiros (Latossolo Vermelho-Amarelo coeso), houve tendência de adensamento dentro dos vasos causado pela acomodação das partículas de argila, o que prejudicou o desenvolvimento das plantas nessa amostra do solo. Em campo, esta é uma das razões pelas quais ocorrem insucessos em cultivos realizados nesse tipo de solo, mesmo com a utilização de subsolagem.

Na SUB, a aplicação da maior dose de calcário reduziu a altura de plantas (TAB. 9 e 10). Esse fato pode estar ligado a um maior adensamento do solo nos vasos, causado por uma maior dispersão da argila a partir da elevada dose de calcário (SPERA et al., 2008), prejudicando ainda mais o crescimento radicular e o crescimento da parte aérea.

Já na SUP, não ocorreram diferenças nas características de desenvolvimento das plantas ao se utilizar uma dose de $0,4 \times$ NC ou de 1,2 x NC (TAB. 9 
e 10), uma vez que a dose básica de calcário calculada foi extremamente baixa $(0,32 \mathrm{t} / \mathrm{ha}$ de calcário).

Utilizando-se a menor dose de calcário na SUB, a matéria seca de raízes foi reduzida linearmente quando se aumentaram as doses de resíduo, sendo que a altura máxima das plantas foi atingida com a dose de 5,4 $\mathrm{tha}^{-1}$ de resíduo de beneficiamento do granito (TAB. 11). Como a dose de calcário de $0,4 \times \mathrm{NC}$ pouco elevou o $\mathrm{pH}$ desta amostra de solo, e ainda ocorreu elevado adensamento do solo nos vasos, essas observações não podem ser extrapoladas com segurança, apesar de significativas.

Por outro lado, utilizando-se a maior dose de calcário na SUB, foram selecionadas equações raiz quadráticas e quadráticas para as características avaliadas, demonstrando que a maior altura de plantas e as maiores produções de matéria seca de raízes e de parte aérea seriam obtidas com 23; 17,8 e 18,2 $\mathrm{t} \mathrm{ha}^{-1}$ de resíduo de beneficiamento do granito, respectivamente (TAB. 11). Em todos os casos, o pH alcançado com essas doses de resíduo seria 5,0 ou muito próximo deste valor (TAB. 5).

Na SUP, quando foi aplicada a menor dose de calcário $(0,4 \times \mathrm{NC})$, foram observados efeitos quadráticos para altura de plantas e matéria seca da parte aérea e efeito linear positivo para matéria seca de raízes ao se aplicarem doses crescentes de resíduo (TAB. 11). Maiores valores de altura de planta e matéria seca da parte aérea foram obtidos com doses de 17,6 e 19,8 t ha ${ }^{-1}$ de resíduo de beneficiamento do granito. Quando a SUP recebeu a maior dose de calcário $(1,2 \times \mathrm{NC})$, os resultados de crescimento observados não mostraram significância ou não foram consistentes (TAB. 11).

As doses selecionadas para a amostra superficial (SUP) e para a amostra subsuperficial do solo (SUB) estão compreendidas no intervalo de doses sugerido por Rosen (2002) para a utilização do pó de granito como fertilizante natural. Esse autor considera que doses entre 15 e $20 \mathrm{t} \mathrm{ha}^{-1}$ de pó de granito sejam suficientes para suprir as plantas com potássio, desde que o tempo necessário para a sua solubilização seja atingido.

Considerando as doses selecionadas no presente estudo, fica claro que o resíduo de beneficiamento do granito não é um material fertilizante de boa qualidade, haja vista que apresenta baixa capacidade de fornecer nutrientes para as plantas, em função de sua reduzida concentração de nutrientes. Assim, sua aplicação em plantios convencionais, que utilizam adubos solúveis, estaria, a princípio, descartada, como sugerido por Rosen (2002).

Em plantios orgânicos, por outro lado, poderia ser utilizado como fertilizante alternativo, uma vez que não traz perigo de contaminação do ambiente com metais pesados e aumenta a produção, apesar das dosagens mais elevadas requeridas. Ainda assim, as doses de resíduo de beneficiamento do granito não seriam maiores do que as doses de outros resíduos, orgânicos ou não, aplicados nessa situação.

\section{Conclusões}

1. A aplicação de doses crescentes do resíduo de beneficiamento do granito elevou o $\mathrm{pH}$ do solo e reduziu o teor de $\mathrm{Al}^{3+}$, de forma linear, mas em pequena magnitude;

2. A utilização do resíduo de beneficiamento do granito foi mais eficiente em amostra de solo com maior capacidade tampão;

3. Para o crescimento inicial do café, o resíduo de beneficiamento do granito deve ser utilizado, exclusivamente, como fonte de $\mathrm{K}$ e $\mathrm{Ca}$, sendo necessário, para isso, que o pH do solo esteja em torno de 5,0;

4. Doses próximas a $20 \mathrm{tha}^{-1}$ do resíduo de beneficiamento do granito geraram crescimento inicial adequado para o café;

5. As plantas de café, durante seu crescimento inicial, não absorveram quantidades expressivas de metais pesados, mesmo aplicando-se doses equivalentes a $30 \mathrm{t} \mathrm{ha}^{-1}$ do resíduo de beneficiamento do granito.

\section{Referências}

ALLOWAY, B. J. Heavy metals in soils. 2. ed. London: Blackie Academic \& Professional, 1995. 368 p.

ALVAREZ V., V. H. et al. Determinação e uso do fósforo remanescente. Boletim Informativo da Sociedade Brasileira de Ciência do Solo, v. 52, n. 01, p. 27-32, 2000.

AMARAL SOBRINHO, N. M. B. et al. Metais pesados em alguns fertilizantes e corretivos. Revista Brasileira de Ciência do Solo, v. 16, n. 02, p. 271-276, 1992.

AMARAL SOBRINHO, N. M. B.; VELlOSO, A. C. X.; OLIVEIRA, C. Solubilidade de metais pesados em solo tratado com resíduo siderúrgico. Revista Brasileira de Ciência do Solo, v. 21, n. 01, p. 9-16, 1997.

BERTON, R. S. Riscos de contaminação do agrossistema com metais pesados. In: BETTIOL, W.; CAMARGO, O. A. (Ed.). Impacto ambiental do uso agrícola do lodo de esgoto. Jaguariúna: Embrapa Meio Ambiente, 2000. p. 259-268.

BOLLAND, M. D. A.; BAKER, M. J. Powdered granite is not an effective fertilizer for clover and wheat in sandy soils from Western Australia. Nutrient Cycling in Agroecosystems, v. 56, n. 01, p. $59-68,2000$. 
CAIRES, E. F. et al. Soil acidity, liming and soybean performance under no-till. Scientia Agricola, v. 65, n. 05, p. 532-540, 2008.

EMPRESABRASILEIRADEPESQUISA AGROPECUÁRIA (EMBRAPA). Manual de métodos de análise de solo. 2. ed. Rio de Janeiro: Centro Nacional de Pesquisa de Solo, 1997. $212 \mathrm{p}$.

GUARÇONI M., A.; ALVAREZ V., V. H.; CAMILO, N. F. P. Avaliação e validação de algoritmo utilizado para determinar a necessidade de calagem em lavouras de café. In: Simpósio de Pesquisa dos Cafés do Brasil, 5., 2007, Águas de Lindóia. Anais... Águas de Lindóia: EMBRAPA, 2007. 1 CD-ROM.

HARLEY, A. D.; GILKES, R. J. Factors influencing the release of plant nutrient elements from silicate rock powders: a geochemical overview. Nutrient Cycling in Agroecosystems, v. 56, n. 01, p. $11-36,2000$.

LANI, J. A.; PREZOTTI, L. C.; BRAGANÇA, S. M. Cafeeiro. In: PREZOTTI, L. C. et al. (Ed.). Manual de Recomendação de Calagem e Adubação para o Estado do Espírito Santo. Vitória: Incaper/SEEA/CEDAGRO, 2007. p. 111-118.

MENEZES, R. R.; FERREIRA, H. S.; NEVES, G. de A. The use of granite wastes as ceramic raw materials. Cerâmica, v. 48, n. 306, p. 92-101, 2002.

NACHTIGALL, G. R; NOGUEIROL, R. C.; ALLEONI, L. R. F. Extração seqüencial de $\mathrm{Mn}$ e $\mathrm{Zn}$ em solos em função do $\mathrm{pH}$ e adição de cama-de-frango. Revista Brasileira de Engenharia Agrícola e Ambiental, v. 13, n. 03, p. 240-249, 2009.

NOGUEIRA, F. D. et al. Respostas de mudas de cafeeiro à aplicação de fosfato natural e ácido cítrico. In: Simpósio de Pesquisa dos Cafés do Brasil, 2., 2001, Vitória. Anais... Vitória: EMBRAPA, 2001. 1 CD-ROM.
OLIVEIRA, G. C. et al. Caracterização química e físico-hídrica de um latossolo vermelho após vinte anos de manejo e cultivo do solo. Revista Brasileira de Ciência do Solo, v. 28, n. 02, p. 327-336, 2004.

RHEINHEIMER, D. S. et al. Dessorção de fósforo avaliada por extrações sucessivas em amostras de solo provenientes dos sistemas plantio direto e convencional. Ciência Rural, v. 33, n. 06, p. 1053-1059, 2003.

ROSEN, C. Agricultural use of rock fines as a sustainable soil amendment. In: MINNESOTA DEPARTMENT OF AGRICULTURE (Ed.). Greenbook. St. Paul: MINNESOTA DEPARTMENT OF AGRICULTURE, 2002. p. 49-51.

TEDESCO, M. J. et al. Análise de solo, plantas e outros materiais. 2. ed. Porto Alegre: UFRGS, Departamento de Solos, 1995. 174 p. (Boletim Técnico, 5).

SPERA, S. T. et al. Dispersão de argila em microagregados de solo incubado com calcário. Revista Brasileira de Ciência do Solo, v. 32, p. 2613-2620, 2008. Número Especial.

VASCONCELOS, A. C. F.; CHAVES, L. H. G.; LUNA, J. G. Uso agrícola da lama de serragem de blocos de granito: efeito na fertilidade do solo. Agropecuária Técnica, v. 24, n. 01, p. 23-30, 2003a.

VASCONCELOS, A. C. F.; CHAVES, L. H. G.; LUNA, J. G. Uso agrícola da lama de serragem de blocos de granito: efeito no crescimento e estado nutricional do milho. Agropecuária Técnica, v. 24, n. 02, p. 139-145, 2003b.

VILLA, M. R.; FERNANDES, L. A.; FAQUIN, V. Formas de potássio em solos de várzea e sua disponibilidade para o feijoeiro. Revista Brasileira de Ciência do Solo, v. 28, n. 04, p. 649-658, 2004. 